\title{
A local graph partitioning algorithm using heat kernel pagerank
}

\author{
Fan Chung \\ University of California at San Diego, La Jolla CA 92093, USA, \\ fan@ucsd.edu, http://www. math.ucsd.edu/ fan/
}

\begin{abstract}
We give an improved local partitioning algorithm using heat kernel pagerank, a modified version of PageRank. For a subset $S$ with Cheeger ratio (or conductance) $h$, we show that there are at least a quarter of the vertices in $S$ that can serve as seeds for heat kernel pagerank which lead to local cuts with Cheeger ratio at most $O(\sqrt{h})$, improving the previously bound by a factor of $\sqrt{\log |S|}$.
\end{abstract}

\section{Introduction}

With the emergence of massive information networks, many previous algorithms are often no longer feasible. A basic setup for a generic algorithm usually includes a graph as a part of its input. This, however, is no longer possible for dealing with massive graphs with prohibitive large size. Instead, the (host) graph, such as the WWW-graph or various social networks, is usually meticulously crawled, organized and stored in some appropriate database. The local algorithms that we study here involve only "local access" of the database of the host graph. For example, getting a neighbor of a specified vertex is considered to be a type of local access. Of course, it is desirable to minimize the number of local accesses needed, hopefully independent of $n$, the number of vertices in the host graph (which may as well be regarded as "infinity"). In this paper, we consider a local algorithm that improves the performance bound of previous local partitioning algorithms.

Graph partitioning problems have long been studied and used for a wide range of applications, typically along the line of divide-and-conquer approaches. Since the exact solution for graph partitioning is known to be NP-complete [12], various approximation algorithms have been utilized. One of the best known partition algorithms is the spectral algorithm. The vertices are ordered by using an eigenvector and only cuts which are initial segments in such an ordering are considered. The advantage of such a "one-sweep" algorithm is to reduce the number of cuts under consideration from an exponential number in $n$ to a linear number. Still, there is a performance guarantee within a quadratic order by using a relation between eigenvalues and the Cheeger constant, called the Cheeger inequality. However, for a large graph (say, with a hundred million vertices), the task of computing an eigenvector is often too costly and not competitive.

A local partitioning algorithm finds a partition that separates a subset of nodes of specified size near specified seeds. In addition, the running time of a local algorithm is required to be proportional to the size of the separated part but independent of the total size of the graph. In [21], Spielman and Teng first gave a local partitioning algorithm using random walks. The analysis of their algorithm is based on a mixing result of Lovász and Simonovits in their work on approximating the volume of convex body. The same mixing result was also proved by Mihail earlier independently [19]. In a previous paper [1], a local partitioning algorithm was given using PageRank, a concept first introduced by Brin and Page [3] in 1998 which has been widely used for Web search algorithms. PageRank is a quantitative ordering of vertices based on random walks on the Webgraph. The notion of PageRank which can be carried out for any graph is basically an efficient way of organizing random walks in a graph. As seen in the detailed definition given later, PageRank can be expressed as a geometric sum of random walks starting from the seed (or an initial probability distribution), with its speed of propagation controlled by a jumping constant. The usual question in random walks is to determine how many steps are required to get close to stationary distribution. In the use of PageRank, the 
problem is reduced to specifying the range for the jumping constant to achieve the desired mixing. The advantage of using PageRank as in [1] is to reduce the computational complexity by a factor of $\log n$.

In this paper, we consider a modified version of PageRank called heat kernel pagerank. Like PageRank, the heat kernel pagerank has two parameters, a seed, and a heat constant or temperature. The heat kernel pagerank can be expressed as an exponential sum of random walks from the seed, scaled by the temperature . In addition, the heat kernel pagerank satisfies a heat equation which dictates the rate of diffusion. We will examine several useful properties of the heat kernel pagerank. In particular, for a given subset of vertices, we consider eigenvalues on the induced subgraph on $S$ satisfying Dirichlet boundary condition (details to be given in the next section). We will show that for a subset $S$ with Cheeger ratio $h$, there are many vertices in $S$ (whose volume is at least a quarter of the volume of $S$ ) such that the one-sweep algorithm using heat kernel pagerank with such vertices as seeds will find local cuts with Cheeger ratio $O(\sqrt{h})$. This improves the previous bound of $O(\sqrt{h \log s})$ in a similar theorem using PageRank [1,2]. Here $s$ denotes the volume of $S$ and a local cut has volume at most $s$.

\section{Preliminaries}

In a graph $G$, the transition probability matrix $W$ of a typical random walk on a graph $G=(V, E)$ is a matrix with columns and rows indexed by $V$ and is defined by :

$$
W(u, v)= \begin{cases}\frac{1}{d_{u}} & \text { if }\{u, v\} \in E, \\ 0 & \text { otherwise }\end{cases}
$$

where the degree of $v$, denoted by $d_{v}$, is the number of vertices that $v$ is adjacent to. We can write $W=D^{-1} A$ where $A$ denotes the adjacency matrix of $G$ and $D$ is the diagonal degree matrix.. A random walk on a graph $G$ has a stationary distribution $\pi$ if $G$ is connected and non-bipartite. The stationary distribution $\pi$, if exists, satisfies $\pi(u)=d_{u} / \sum_{v} d_{v}$.

The PageRank we consider here is also called personalized PageRank (see $[13,14]$ ), which generalized the version first introduced by Brin and Page [3]. PageRank has two parameters, a preference vector $f$ (i.e., the probabilistic distribution of the seed(s)) and a jumping constant $\alpha$. Here, the function $f: V \rightarrow \mathbb{R}$ is taken to be a row vector so that $W$ can act on $f$ from the right by matrix multiplication. The PageRank $\operatorname{pr}_{\alpha, f}$, with the scale parameter $\alpha$ and the preference vector $f$, satisfies the following recurrence relation:

$$
\operatorname{pr}_{\alpha, f}=\alpha f+(1-\alpha) \operatorname{pr}_{\alpha, f} W .
$$

An equivalent definition for PageRank is the following:

$$
\operatorname{pr}_{\alpha, f}=\alpha \sum_{k=0}^{\infty}(1-\alpha)^{k} f W^{k} .
$$

For example, if we have one starting seed denoted by vertex $u$, then $f$ can be written as the $(0,1)$-indicator function $\chi_{u}$ of $u$. Another example is to take $f$ to be the constant function with value $1 / n$ at every vertex as in the original definition of PageRank in Brin and Page [3].

The heat kernel pagerank also has two parameters, with (the temperature) $t \geq 0$ and a preference vector $f$, defined as follows:

$$
\rho_{t, f}=e^{-t} \sum_{k=0}^{\infty} \frac{t^{k}}{k !} f W^{k} .
$$


We see that (3) is just an exponential sum versus (2) is a geometrical sum. For many combinatorial problems, exponential generating functions play a useful role. The heat kernel pagerank as defined in (3) satisfies the following heat equation:

$$
\frac{\partial}{\partial t} \rho_{t, f}=-\rho_{t, f}(I-W)
$$

Let us define $L=I-W$. Then the definition of the heat kernel pagerank in (3) can be rewritten as follows:

$$
\rho_{t, f}=f H_{t},
$$

where $H_{t}$ is defined by

$$
\begin{aligned}
H_{t} & =e^{-t} \sum_{k=0}^{\infty} \frac{t^{k}}{k !} W^{k} \\
& =e^{-t(I-W)} \\
& =e^{-t L} \\
& =\sum_{k=0}^{\infty} \frac{(-t)^{k}}{k !} L^{k} .
\end{aligned}
$$

From the above definition, we have the following facts for $\rho_{t, f}$ which will be useful later.

Lemma 1. For a graph $G$, its heat kernel pagerank $\rho$ satisfies the following:

(i) $\rho_{0, f}=f$.

(ii) $\rho_{t, \pi}=\pi$.

(iii) $\rho_{t, f} \mathbf{1}^{*}=f \mathbf{1}^{*}=1$ if $f$ satisfies $\sum_{v} f(v)=1$ where $\mathbf{1}$ denotes the all 1 's function (as a row vector) and $\mathbf{1}^{*}$ denotes the transpose of $\mathbf{1}$.

(iv) $W H_{t}=H_{t} W$.

(v) $D H_{t}=H_{t}^{*} D$ and $H_{t}=H_{t / 2} H_{t / 2}=H_{t / 2} D^{-1} H_{t / 2}^{*} D$.

Proof. Since $H_{0}=I$, (i) follows. (ii) and (iii) can be easily checked. (iv) follows from the fact that $H_{t}$ is a polynomial of $W$. (v) is a consequence of the fact that $W=D^{-1} A$.

\section{Dirichlet eigenvalues and the restricted heat kernel}

For a subset $S$ of $V(G)$, there are two types of boundary of $S$ - the vertex boundary $\delta(S)$ and edge boundary $\partial(S)$. The vertex boundary $\delta(S)$ is defined as follows:

$$
\delta(S)=\{u \in V(G) \backslash S: u \sim v \text { for some } v \in S\} .
$$

For a single vertex $v$, the degree of $v$, denoted by $d_{v}$ is equal to $|\delta(v)|$ (which is short for $|\delta(\{v\})|$.

The closure of $S$, denoted by $S^{*}$, is the union of $S$ and $\delta S$. For a function $f: S^{*} \rightarrow \mathbb{R}$, we say $f$ satisfies the Dirichlet boundary condition if $f(u)=0$ for all $u \in \delta(S)$. We use the notation $f \in \mathbf{D}_{S}^{*}$ to denote that $f$ satisfies the Dirichlet boundary condition and we require that $f \neq 0$. 
For $f \in \mathbf{D}_{S}^{*}$, we define a Dirichlet Rayleigh quotient:

$$
R_{S}(f)=\frac{\sum_{x \sim y}(f(x)-f(y))^{2}}{\sum_{x \in S} f^{2}(x) d_{x}} .
$$

where the sum is to be taken over all unordered pairs of vertices $x, y \in S^{*}$ such that $s \sim y$.

The Dirichlet eigenvalue of an induced subgraph on $S$ of a graph $G$ can be defined as follows:

$$
\begin{aligned}
\lambda_{S} & =\inf _{f \in \mathbf{D}_{S}^{*}} R_{S}(f) \\
& =\inf _{f \in \mathbf{D}_{S}^{*}} \frac{\left\langle f,\left(D_{S}-A_{S}\right) f\right\rangle}{\left\langle f, D_{S} f\right\rangle} \\
& =\inf _{g \in \mathbf{D}_{S}^{*}} \frac{\left\langle g, \mathcal{L}_{S} g\right\rangle}{\langle g, g\rangle},
\end{aligned}
$$

where $X_{S}$ denotes the submatrix of a matrix $X$ with rows and columns restricted to those indexed by vertices in $S$, and the Laplacian $\mathcal{L}$ and $\mathcal{L}_{S}$ are defined by $\mathcal{L}=D^{-1 / 2}(D-A) D^{-1 / 2}$ and $\mathcal{L}_{S}=D_{S}^{-1 / 2}\left(D_{S}-\right.$ $\left.A_{S}\right) D_{S}^{-1 / 2}$, respectively. Here we call $f$ the combinatorial Dirichlet eigenfunction if $R_{S}(f)=\lambda_{S}$. The Dirichlet eigenfunctions are the eigenfuctions of the matrix $\mathcal{L}_{S}$. (The detailed proof of these statements can be found in $[5]$.

The Dirichlet eigenvalues of $S$ are the eigenvalues of $\mathcal{L}_{S}$, denoted by

$$
\lambda_{S, 1} \leq \lambda_{S, 2} \leq \cdots \leq \lambda_{S, s}
$$

where $s=|S|$. The smallest Dirichlet eigenvalue $\lambda_{S, 1}$ is also denoted by $\lambda_{S}$. If the induced subgraph on $S$ is connected, then the eigenvector of $\mathcal{L}_{S}$ associated with $\lambda_{S}$ is all positive (using the Perron-Frobenius Theorem [20] on $I-\mathcal{L}_{S}$ ). The reader is referred to [5] for various properties of Dirichlet eigenvalues.

For a subset $S$ of vertices in $G$, the edge separater (or the edge boundary) whose removal separates $S$ consists of all edges leaving $S$. Namely,

$$
\partial S=\{\{u, v\} \in E: u \in S \text { and } v \notin S\} .
$$

How good is the edge separator? The answer depends both on the size of the edge separator and the volume of $S$, denoted by $\operatorname{vol}(S)$, is $\sum_{u \in S} d_{u}$. The volume of a graph $G$, denoted by $\operatorname{vol}(G)$, is $\sum_{u} d_{u}$.

The Cheeger ratio of $S$, denoted by $h_{S}$, is defined by

$$
h_{S}=\frac{|\partial S|}{\min \{\operatorname{vol}(S), \operatorname{vol}(\bar{S})\}}
$$

where $\bar{S}=V \backslash S$ denotes the complement of $S$. The Cheeger constant of a graph $G$ is $h_{G}=\min _{S \subset V} h_{S}$. The Cheeger constant of a graph $G$ is often called the conductance of $G$. For a given subset $S$, we define the local Cheeger ratio, denoted by $h_{S}^{*}$, as follows:

$$
h_{S}^{*}=\inf _{T \subseteq S} h_{T} .
$$

Note that in general $h_{S}$ is not necessarily equal to $h_{S}^{*}$.

The Dirichlet eigenvalue $\lambda_{S}$ and the local Cheeger ratio $h_{S}^{*}$ are related by the following local Cheeger inequality:

$$
h_{S}^{*} \geq \lambda_{S} \geq \frac{\left(h_{S}^{*}\right)^{2}}{2},
$$


while the proof can be found in [6].

In [8], the following weighted Rayleigh quotient:

$$
R_{\phi}(f)=\sup _{c} \frac{\sum_{x \sim y}(f(x)-f(y))^{2} \phi(x) \phi(y)}{\sum_{x \in S}(f(x)-c)^{2} \phi^{2}(x) d_{x}},
$$

where $\phi$ is the combinatorial Dirichlet eigenfunction which achieves $\lambda_{S}$. Then we can define

$$
\lambda_{\phi}=\inf _{f \neq 0} R_{\phi}(f) .
$$

Here we state several useful facts concerning $\lambda_{S}$ and $\lambda_{\phi}$ (see [8]).

Lemma 2. For an induced subgraph $S$ of a graph $G$, the Dirichlet eigenvalues of $S$ satisfy

$$
\lambda_{S, 2}-\lambda_{S}=\lambda_{\phi} \geq \lambda_{S}
$$

Theorem 1. For an induced subgraph $S$ of $G$, the combinatorial Dirichlet eigenfunction $\phi$ which achieves $\lambda_{S}$ satisfies

$$
\left(\sum_{x \in S} \phi(x) d_{x}\right)^{2} \geq \frac{1}{2} \operatorname{vol}(S) \sum_{x \in S} \phi^{2}(x) d_{x} .
$$

Proof. We note that

$$
\lambda_{S, 2}=\inf _{f} \frac{\sum_{x \sim y}(f(x)-f(y))^{2}}{\sum_{x \in S} f(x)^{2} d_{x}},
$$

where $f$ ranges over all functions satisfying $\sum_{x \in S} f(x) \phi(x)=0$. Now we use the fact that

$$
\lambda_{S}=\frac{\sum_{x \sim y}(\phi(x)-\phi(y))^{2}}{\sum_{x \in S} \phi(x)^{2} d_{x}} .
$$

We then have

$$
\begin{aligned}
\lambda_{S, 2} & \leq \frac{\sum_{x \sim y}(\phi(x)-\phi(y))^{2}}{\sum_{x \in S}(\phi(x)-c)^{2} d_{x}} \\
& =\frac{\lambda_{S} \sum_{x \in S} \phi^{2}(x) d_{x}}{\sum_{x \in S} \phi^{2}(x) d_{x}-c^{2} \operatorname{vol}(S)},
\end{aligned}
$$

where

$$
c=\frac{\sum_{x \in S} \phi(x) d_{x}}{\operatorname{vol}(S)}
$$

This implies

$$
\begin{aligned}
\frac{\left(\sum_{x \in S} \phi(x) d_{x}\right)^{2}}{\operatorname{vol}(S) \sum_{x \in S} \phi^{2}(x) d_{x}} & \geq \frac{\lambda_{S, 2}-\lambda_{S}}{\lambda_{S, 2}} \\
& =\frac{\lambda_{\phi}}{\lambda_{\phi}+\lambda_{S}} \\
& \geq \frac{1}{2}
\end{aligned}
$$

by using Lemma 2 . 


\section{A lower bound for the restricted heat kernel pagerank}

For a given set $S$, we consider the distribution $f_{S}$ with choosing the vertex $u$ with probability $f_{S}(u)=$ $d_{u} / \operatorname{vol}(S)$ if $u \in S$, and 0 otherwise. Note that $f_{S}$ can be written as $\frac{1}{\operatorname{vol}(S)} \chi_{S} D$ where $\chi_{S}$ is the indicator function for $S$. For any function $g: V \rightarrow \mathbb{R}$, we define $g(S)=\sum_{v \in S} g(v)$.

In this section, we wish to establish a lower bound for the expected value of heat kernel pagerank $\rho_{t, u}=\rho_{t, \chi_{u}}$ over $u$ in $S$. We note that

$$
\begin{aligned}
\mathbb{E}\left(\rho_{t, u}\right) & =\sum_{u \in S} \frac{d_{u}}{\operatorname{vol}(S)} \rho_{t, u} \\
& =f_{S} H_{t}(S)
\end{aligned}
$$

We consider the restricted heat kernel $H_{t}^{\prime}$ for a fixed subset $S$, defined as follows: Then the definition of the heat kernel pagerank in (3) can be rewritten as follows:

$$
\rho_{t, f}^{\prime}=f H_{t}^{\prime}
$$

where $H_{t}^{\prime}$ is defined by

$$
\begin{aligned}
H_{t}^{\prime} & =e^{-t} \sum_{k=0}^{\infty} \frac{t^{k}}{k !} W_{S}^{k} \\
& =e^{-t\left(I_{S}-W_{S}\right)} \\
& =e^{-t L_{S}} \\
& =\sum_{k=0}^{\infty} \frac{(-t)^{k}}{k !} L_{S}^{k}
\end{aligned}
$$

Also, $H_{t}^{\prime}$ satisfies the following heat equation:

$$
\frac{\partial}{\partial t} H_{t}^{\prime}=-L_{S} H_{t}^{\prime}
$$

From the above definition, we immediately have the following:

\section{Lemma 3.}

$$
H_{t}^{\prime}(x, y) \leq H_{t}(x, y)
$$

for all vertices $x$ and $y$. In particular, for a non-negative function $f: V \rightarrow \mathbb{R}$, we have

$$
f H_{t}^{\prime}(v) \leq f H_{t}(v)
$$

for every vertex $v$ in $G$.

Therefore, it suffices to establish the desired lower bound for the expected value of restricted heat kernel pagerank.

Following the notation in Section 3, we consider the Dirichlet eigenvalues $\lambda_{S, i}$ of $S$ and their associated Dirichlet combinatorial eigenfunction $\phi_{i}$ with $R\left(\phi_{i}\right)=\lambda_{S, i}$, for $i=1, \ldots,|S|$. Clearly, $\phi_{i} D_{S}^{1 / 2}$ are orthogonal 
eigenfunction of $\mathcal{L}_{S}$ and form a basis for functions defined on $S$. Here we assume that $\sum_{u \in S} \phi_{i}(u)^{2} d_{u}=1$. To simplify the notation, in this proof we write $\lambda_{i}^{\prime}=\lambda_{S, i}$ and $\lambda_{1}^{\prime}=\lambda_{S}$.

We express $f=\sqrt{\operatorname{vol}(S)} f_{S}$ in terms of $\phi_{i}$ as follows:

$$
f D_{S}^{-1 / 2}=\sum_{i} a_{i} \phi_{i} D_{S}^{1 / 2}
$$

where

$$
a_{i}=\sum_{u \in S} \frac{\phi_{i}(u) d_{u}}{\sqrt{\operatorname{vol}(S)}}
$$

Since $\left\|f D_{S}^{-1 / 2}\right\|=\left\|f D_{S}^{-1 / 2}\right\|_{2}=1$, we have

$$
\sum_{i} a_{i}^{2}=1
$$

From the above definitions we have

$$
f_{S} H_{t}^{\prime}(S)=\left\|f \mathcal{H}_{t / 2}^{\prime}\right\|^{2}
$$

where $\mathcal{H}_{t}^{\prime}=D_{S}^{1 / 2} H_{t}^{\prime} D_{S}^{-1 / 2}$. From Theorem 1 , we know the fact that

$$
\begin{aligned}
a_{1}^{2} & =\frac{\left(\sum_{u \in S} \phi_{1}(u) d_{u}\right)^{2}}{\operatorname{vol}(S)} \\
& \geq 1 / 2 \\
& \geq 1-\sum_{j \neq 1} a_{j}^{2} .
\end{aligned}
$$

Since

$$
f_{S} H_{t}^{\prime}(S)=\sum_{i} a_{i}^{2} e^{-\lambda_{i}^{\prime} t}
$$

we have

$$
\begin{aligned}
f_{S} H_{t}^{\prime}(S) & \geq a_{1}^{2} e^{-\lambda_{S} t} \\
& \geq \frac{1}{2} e^{-\lambda_{S} t} .
\end{aligned}
$$

We have proved the following:

Theorem 2. For a subset $S$, the Dirichlet heat kernel $H_{t}^{\prime}$ satisfies

$$
f_{S} H_{t}^{\prime}(S) \geq \frac{1}{2} e^{-\lambda_{S} t}
$$

As an immediate consequence of Theorem 1, Lemmas 2 and 3 and Theorem 2, we have the following:

Corollary 1. In a graph $G$, for a subset $S$ of vertices the heat kernel pagerank $\rho_{t, f_{S}}$ satisfies

$$
\mathbb{E}\left(\rho_{t, u}(S)\right)=\rho_{t, f_{S}}(S) \geq \frac{1}{2} e^{-\lambda_{S} t} \geq \frac{1}{2} e^{-h_{S}^{*} t},
$$

where $u$ is chosen according to $f_{S}$. 


\section{$5 \quad$ A local lower bound for heat kernel pagerank}

Corollary 1 states that the expected value of $\rho_{t, u}$ is at least $e^{-h(S) t}$. Thus, there exists a vertex $u$ in $S$ such that $\rho_{t, u}(S)$ is at least $e^{-h(S) t}$. However, in order to have an efficient local partitioning algorithm, we need to show that there are many vertices $v$ satisfying

$$
\rho_{t, v}(S) \geq c \rho_{t, f_{S}}(S)
$$

for some absolute constant $c$. To do so, we will prove the following:

Theorem 3. In a graph $G$ with a given subset $S$ of vertices, the subset $T=\left\{u \in S: \rho_{t, u}(S) \geq \frac{1}{4} e^{-t \lambda_{S}}\right\}$ satisfies

$$
\operatorname{vol}(T) \geq \frac{1}{4} \operatorname{vol}(S)
$$

if $t \geq 1 / \lambda_{S}$.

From Lemma 3, Theorem 3 follows directly from Theorem 4:

Theorem 4. In a graph $G$ with a given subset $S$ of vertices, the subset $T=\left\{u \in S: \rho_{t, u}^{\prime}(S) \geq \frac{1}{4} e^{-t \lambda_{S}}\right\}$ satisfies

$$
\operatorname{vol}(T) \geq \frac{1}{4} \operatorname{vol}(S)
$$

if $t \geq 1 / \lambda_{S}$.

To prove Theorem 4, we first prove the following lemma along the second-moment methods:

Lemma 4. If $t \geq 1 / \lambda_{S}$, then

$$
\sum_{u \in S} \frac{d_{u}}{\operatorname{vol}(S)}\left(\rho_{t, u}^{\prime}(S)-\rho_{t, f_{S}}^{\prime}(S)\right)^{2} \leq \frac{5}{4} \rho_{t, f_{S}}^{\prime}(S)^{2}
$$

Proof. We note that

$$
\begin{aligned}
\sum_{u \in S} \frac{d_{u}}{\operatorname{vol}(S)}\left(\rho_{t, u}^{\prime}(S)-\rho_{t, f_{S}}^{\prime}(S)\right)^{2} & =\sum_{u \in S} \frac{d_{u}}{\operatorname{vol}(S)} \rho_{t, u}^{\prime}(S)^{2}-\left(\rho_{t, f_{S}}^{\prime}(S)\right)^{2} \\
& =f_{S} H_{2 t}^{\prime}(S)-\left(f_{S} H_{t}^{\prime}(S)\right)^{2}
\end{aligned}
$$

It suffices to show that

$$
f_{S} H_{2 t}^{\prime}(S) \leq \frac{9}{4}\left(f_{S} H_{t}^{\prime}(S)\right)^{2}
$$

We consider the Dirichlet eigenvalues $\lambda_{S, i}$ of $S$ and their associated Dirichlet combinatorial eigenfunctions $\phi_{i}$ with $R\left(\phi_{i}\right)=\lambda_{S, i}=\lambda_{i}^{\prime}$, for $i=1, \ldots,|S|$. Here, $\phi_{i} D_{S}^{1 / 2}$ are orthonormal eigenfunctions of $\mathcal{L}_{S}$ with $\sum_{u \in S} \phi_{i}(u)^{2} d_{u}=1$.

We can write $f=\chi_{S} D_{S} \operatorname{vol}(S)^{-1 / 2}=f_{S} \sqrt{\operatorname{vol}(S)}$ by:

$$
f D_{S}^{-1 / 2}=\sum_{i} a_{i} \phi_{i} D_{S}^{1 / 2}
$$


We have

$$
\sum_{i} a_{i}^{2}=1
$$

since $\left\|f D_{S}^{-1 / 2}\right\|_{2}=1$. From Theorem 1 , we know the fact that

$$
a_{1}^{2} \geq 1 / 2 \geq 1-\sum_{j \neq 1} a_{j}^{2}
$$

Also we have

$$
f_{S} H_{2 t}^{\prime}(S)=\left\|f \mathcal{H}_{t}^{\prime}\right\|^{2}=\sum_{i} a_{i}^{2} e^{-2 \lambda_{i}^{\prime} t}
$$

where $\mathcal{H}_{t}^{\prime}=D_{S}^{1 / 2} H_{t}^{\prime} D_{S}^{-1 / 2}$.

Therefore we have, for $t \geq 1 / \lambda_{1}^{\prime}$,

$$
\begin{aligned}
f_{S} H_{2 t}^{\prime}(S) & =\sum_{i} a_{i}^{2} e^{-2 \lambda_{i}^{\prime} t} \\
& \leq a_{1}^{2} e^{-2 \lambda_{1}^{\prime} t}+\left(1-a_{1}^{2}\right) e^{-2 \lambda_{2}^{\prime} t} \\
& =a_{1}^{2} e^{-2 \lambda_{1}^{\prime} t}+\left(1-a_{1}^{2}\right) e^{-4 \lambda_{1}^{\prime} t} \\
& \leq \frac{9}{8} a_{1}^{2} e^{-2 \lambda_{1}^{\prime} t}
\end{aligned}
$$

since $\lambda_{2}^{\prime} \geq 2 \lambda_{1}^{\prime}$ by using Lemma 2 . Therefore we have

$$
\begin{aligned}
f_{S} H_{2 t}^{\prime}(S) & \leq \frac{9}{8} a_{1}^{2} e^{-2 \lambda_{S} t} \\
& \leq \frac{9}{4} a_{1}^{4} e^{-2 \lambda_{S} t} \\
& \leq \frac{9}{4}\left(\sum_{i} a_{i}^{2} e^{-\lambda_{i}^{\prime} t}\right)^{2}
\end{aligned}
$$

as desired.

Now, we are ready to proceed to prove Theorem 4 which then implies Theorem 3 .

Proof of Theorem 4:

Suppose $\operatorname{vol}(T) \leq \operatorname{vol}(S) / 4$. We wish to show that this leads to a contradiction. Let $T^{\prime}=S \backslash T$. We consider

$$
\begin{aligned}
& \sum_{u \in S} \frac{d_{u}}{\operatorname{vol}(S)}\left(\rho_{t, u}^{\prime}(S)-\rho_{t, f_{S}}^{\prime}(S)\right)^{2} \\
= & \sum_{u \in T} \frac{d_{u}}{\operatorname{vol}(S)}\left(\rho_{t, u}^{\prime}(S)-\rho_{t, f_{S}}^{\prime}(S)\right)^{2}+\sum_{u \in T^{\prime}} \frac{d_{u}}{\operatorname{vol}(S)}\left(\rho_{t, u}^{\prime}(S)-\rho_{t, f_{S}}^{\prime}(S)\right)^{2} \\
\geq & \frac{\left(\sum_{u \in T} \frac{d_{u}}{\operatorname{vol}(S)}\left(\rho_{t, u}^{\prime}(S)-\rho_{t, f_{S}}^{\prime}(S)\right)\right)^{2}}{\frac{\operatorname{vol}(T)}{\operatorname{vol}(S)}}+\frac{\left(\sum_{u \in T^{\prime}} \frac{d_{u}}{\operatorname{vol}(S)}\left(\rho_{t, u}^{\prime}(S)-\rho_{t, f_{S}}^{\prime}(S)\right)\right)^{2}}{\frac{\operatorname{vol}\left(T^{\prime}\right)}{\operatorname{vol}(S)}} \\
\geq & \left(\sum_{u \in T^{\prime}} \frac{d_{u}}{\operatorname{vol}(S)}\left(\rho_{t, u}^{\prime}(S)-\rho_{t, f_{S}}^{\prime}(S)\right)\right)^{2}\left(\frac{1}{\frac{\operatorname{vol}(T)}{\operatorname{vol}(S)}}+\frac{1}{\frac{\operatorname{vol}\left(T^{\prime}\right)}{\operatorname{vol}(S)}}\right)
\end{aligned}
$$

since

$$
\sum_{u \in S} \frac{d_{u}}{\operatorname{vol}(S)}\left(\rho_{t, u}^{\prime}-\rho_{t, f_{S}}^{\prime}\right)=0 .
$$


Therefore we have

$$
\begin{aligned}
\sum_{u \in S} \frac{d_{u}}{\operatorname{vol}(S)}\left(\rho_{t, u}^{\prime}(S)-\rho_{t, f_{S}}^{\prime}(S)\right)^{2} & \geq\left(\frac{\operatorname{vol}\left(T^{\prime}\right)}{\operatorname{vol}(S)}\left(\frac{3}{4}\right) \rho_{t, f_{S}}^{\prime}(S)\right)^{2}\left(\frac{1}{\frac{\operatorname{vol}(T)}{\operatorname{vol}(S)}}+\frac{1}{\frac{\operatorname{vol}\left(T^{\prime}\right)}{\operatorname{vol}(S)}}\right) \\
& \geq\left(4\left(\frac{3}{4}\right)^{4}+\left(\frac{3}{4}\right)^{3}\right) \rho_{t, f_{S}}^{\prime}(S)^{2} \\
& \geq \frac{27}{16} \rho_{t, f_{S}}^{\prime}(S)^{2} \\
& >\frac{5}{4} \rho_{t, f_{S}}^{\prime}(S)^{2}
\end{aligned}
$$

which is a contradiction to Lemma 4 . Theorem 4 is proved.

\section{An upper bound for heat kernel pagerank}

For a function $f: V \rightarrow \mathbb{R}$, we can order vertices according to their $f$ values. The ordered list is then called a sweep. The segment $S_{i}$ consists of the first $i$ vertices (by breaking ties arbitrarily). We define a $s$-local Cheeger ratio of a sweep $f$, denoted by $h_{f, s}$ to be the minimum Cheeger ratio of the segment $S_{i}$ with $0 \leq \operatorname{vol}\left(S_{i}\right) \leq 2 s$. If no such segment exists, then we set $h_{f, s}$ to be 0 . We will establish the following upper bound for the heat kernel pagerank in terms of $s$-local Cheeger ratios. The proof is similar but simpler than that in [7].

Theorem 5. In a graph $G$ with a subset $S$ with volume $s \leq \operatorname{vol}(G) / 4$, for any vertex $u$ in $G$, we have

$$
\rho_{t, u}(S)-\pi(S) \leq \sqrt{\frac{s}{d_{u}}} e^{-t \kappa_{t, u, s}^{2} / 4}
$$

where $\kappa_{t, u, s}$ denote the minimum s-local Cheeger ratio over a sweep of $\rho_{t, u}$.

Proof. For a function $f: V \rightarrow \mathbb{R}$, we define $f(u, v)=f(u) / d_{u}$ if $v$ is adjacent to $u$ and 0 otherwise. For an integer $x, 0 \leq x \leq \operatorname{vol}(G) / 2$, we define

$$
f(x)=\max _{T \subseteq V \times V,|T|=x} \sum_{(u, v) \in T} f(u, v) .
$$

We can extend $f$ to all real $x=k+r$, with $0 \leq r<1$ by defining $f(x)=(1-r) f(k)+r f(k+1)$. If $x=\operatorname{vol}\left(S_{i}\right)$ where $S_{i}$ consists of vertices with the $i$ highest values of $f(u) / d_{u}$, then it follows from the definition that $f(x)=\sum_{u \in S_{i}} f(u)$. Also $f(x)$ is concave in $x$.

We consider the lazy walk $\mathbf{W}=(I+W) / 2$. Then

$$
\begin{aligned}
f \mathbf{W}(S) & =\frac{1}{2}\left(f(S)+\sum_{u \sim v \in S} f(u, v)\right) \\
& =\frac{1}{2}\left(\sum_{u \text { or } v \in S} f(u, v)+\sum_{u \text { and } v \in S} f(u, v)\right) \\
& \leq \frac{1}{2}(f(\operatorname{vol}(S)+|\partial S|)+f(\operatorname{vol}(S)-|\partial S|)) \\
& =\frac{1}{2}\left(f\left(\operatorname{vol}(S)\left(1+h_{S}\right)\right)+f\left(\operatorname{vol}(S)\left(1-h_{S}\right)\right)\right)
\end{aligned}
$$


This can be straightforwardly extended to real $x$ with $0 \leq x \leq \operatorname{vol}(G) / 2$. In particular, we focus on $x$ satisfying $0 \leq x \leq 2 s \leq \operatorname{vol}(G) / 2$ and we choose $f_{t}=\rho_{t, u}-\pi$. Then

$$
f_{t} \mathbf{W}(x) \leq \frac{1}{2}\left(f_{t}\left(x\left(1+\kappa_{t, u, s}\right)\right)+f_{t}\left(x\left(1-\kappa_{t, u, s}\right)\right)\right) .
$$

We now consider for $x \in[0,2 s]$,

$$
\begin{aligned}
\frac{\partial}{\partial t} f_{t}(x) & =-\rho_{t, u}(I-W)(x) \\
& =-2 \rho_{t, u}(I-\mathbf{W})(x) \\
& =-2 f_{t}(x)+2 f_{t} \mathbf{W}(x) \\
& \leq-2 f_{t}(x)+f_{t}\left(x\left(1+\kappa_{t, u, s}\right)\right)+f_{t}\left(x\left(1-\kappa_{t, u, s}\right)\right) \\
& \leq 0
\end{aligned}
$$

by the concavity of $f_{t}$. Suppose $g_{t}(x)$ is a solution of the equation in (8) satisfying $f_{0}(x) \leq g_{0}(x), f_{t}(0)=g_{t}(0)$ and $\left.\frac{\partial}{\partial t} f_{t}(x)\right|_{t=0} \leq\left.\frac{\partial}{\partial t} g_{t}(x)\right|_{t=0}$. Then, we have $f_{t}(x) \geq g_{t}(x)$. It is easy to check that $g_{t}(x) \leq e^{-t \kappa_{t, u, s}^{2} / 4} \sqrt{\frac{x}{d_{u}}}$ using $-2+\sqrt{1+x}+\sqrt{1-x} \leq-x^{2} / 4$. Thus,

$$
\begin{aligned}
\rho_{t, u}(S)-\pi(S) & \leq \rho_{t, u}(s)-\pi(s) \\
& \leq \sqrt{\frac{s}{d_{u}}} e^{-t \kappa_{t, u, s}^{2} / 4},
\end{aligned}
$$

as desired.

\section{A local Cheeger inequality and a local partitioning algorithm}

Let $h_{s}$ denote the minimum Cheeger ratio $h_{S}$ with $0 \leq \operatorname{vol}(S) \leq 2 s$. Also let $\kappa_{t, 2 s}$ denotes the minimum of $\kappa_{t, u, 2 s}$ over all $u$. Combining Theorem 3 and Theorem 5 , we have that the set of $u$ satisfying

$$
\frac{1}{2} e^{-t h_{S}^{*}} \leq \frac{1}{2} e^{-t \lambda_{S}} \leq \rho_{t, f_{S}}(s)-\pi(s) \leq \sqrt{s} e^{-t \kappa_{t, u, 2 s}^{2} / 4},
$$

has volume at least $\operatorname{vol}(S) / 4$, provided $t \geq 1 / h_{S}^{2} \geq 1 / \lambda_{S}$.

As an immediate consequence, we have the following local Cheeger inequality:

Theorem 6. For a subset $S$ of a graph $G$ with $\operatorname{vol}(S)=s \leq \operatorname{vol}(G) / 4$ and $t \geq \log s /\left(h_{S}^{*}\right)^{2}$, with probability at least $1 / 4$ a vertex $u$ in $S$ satisfies

$$
h_{S}^{*} \geq \lambda_{S} \geq \frac{\kappa_{t, u}^{2}}{4}-\frac{2 \log s}{t}
$$

where the Cheeger ratio $\kappa_{t, u}$ is determined by the heat kernel pagerank with seed $u$.

Corollary 2. For $s \leq \operatorname{vol}(G) / 4$, and $t \geq 4 \log s /\left(h_{S}\right)^{2}$, and a set $S$ of volume $s$, the Cheeger ratio $\kappa_{t, u}$, determined by the heat kernel pagerank with a random seed $u$ in $S$, satisfies

$$
h_{S}^{*} \geq \lambda_{S} \geq \frac{\kappa_{t, u}^{2}}{8}
$$

with probability at least $1 / 4$. 
The above local Cheeger inequalities are closely associated with local partition algorithms. A local partition algorithm has inputs including a vertex as the seed, the volume $s$ of the target set and a target value $\phi$ for the Cheeger ratio of the target set. The local Cheeger inequality in Theorem 5 suggests the following local partition algorithm. In order to find the set achieving the minimum $s$-local Cheeger ratio, one can simply consider a sweep of heat kernel pagerank with further restrictions to the cuts with smaller parts of volume between 0 and $2 s$.

Theorem 6 implies the following.

Theorem 7. In a graph $G$, for a set $S$ with volume $s \leq \operatorname{vol}(G) / 4$, and Cheeger ratio $h_{S} \leq \phi^{2}$, there is a subset $S^{\prime} \subseteq S$ with $\operatorname{vol}\left(S^{\prime}\right) \geq \operatorname{vol}(S) / 4$ such that for any $u \in S^{\prime}$, the sweep by using the heat kernel pagerank $\rho_{t, u}$, with $t=2 \phi^{-2} \log s$, will find a set $T$ with s-local Cheeger ratio at most $2 \phi$.

We note that the performance bound for the Cheeger ratio improves the earlier result in [1] by a factor of $\log s$. In fact, the inequality in Theorem 6 suggests a whole range of trade-off. If we choose $t$ to be $t=2 \phi^{-2}$ instead, then the guaranteed Cheeger ratio as in the above statement will be $2 \phi \log s$ and we obtain the same approximation results as in [1].

We remark that the computational complexity of the above partitioning algorithm is the same as that of computing the heat kernel pagerank. However, the algorithmic design for heat kernel pagerank has not been as extensively studied as the PageRank. More research is needed in this direction.

\section{References}

1. R. Andersen, F. Chung and K. Lang, Local graph partitioning using pagerank vectors, Proceedings of the 47 th Annual IEEE Symposium on Founation of Computer Science (FOCS'2006), 475-486.

2. R. Andersen, F. Chung and K. Lang, Detecting sharp drops in PageRank and a simplified local partitioning algorithm, Theory and Applications of Models of Computation, Proceedings of TAMC 2007, 1-12.

3. S. Brin and L. Page, The anatomy of a large-scale hypertextual Web search engine, Computer Networks and ISDN Systems, 30 (1-7), (1998), 107-117.

4. J. Cheeger, A lower bound for the smallest eigenvalue of the Laplacian, Problems in Analysis (R. C. Gunning, ed.), Princeton Univ. Press (1970), 195-199.

5. F. Chung, Spectral Graph Theory, AMS Publications, 1997.

6. F. Chung, Random walks and local cuts in graphs, LAA 423 (2007), 22-32.

7. F. Chung, The heat kernel as the pagerank of a graph, PNAS, 105 (50), (2007), 19735-19740.

8. F. Chung and K. Oden, Weighted graph Laplacians and isoperimetric inequalities, Pacific Journal of Mathematics 192 (2000), 257-273.

9. F. Chung and S.-T. Yau, Coverings, heat kernels and spanning trees, Electronic Journal of Combinatorics 6 (1999), \#R12.

10. F. Chung and L. Lu, Complex Graphs and Networks, CBMS Regional Conference Series in Mathematics, 107, AMS Publications, RI, 2006. viii+264 pp.

11. D. Coppersmith and S. Winograd, Matrix multiplication via arithmetic progressions, J. Symbolic Comput. 9 (1990), 251-280.

12. M. R. Garey and D. S. Johnson, Computers and Intractability. A Guide to the theory of NP-completeness, W. H. Freeman, San Francisco, 1979, x+338 pp.

13. T. H. Haveliwala. Topic-sensitive pagerank: A context-sensitive ranking algorithm for web search, IEEE Trans. Knowl. Data Eng., 15, no. 4, (2003), 784796.

14. G. Jeh and J. Widom, Scaling personalized web search, Proceedings of the 12th World Wide Web Conference $(W W W)(2003), 271279$.

15. M. Jerrum and A. J. Sinclair, Approximating the permanent, SIAM J. Computing 18 (1989), 1149-1178.

16. R. Kannan and S. Vempala and A. Vetta, On clusterings: Good, bad and spectral, JACM 51 (2004), 497-515.

17. L. Lovász and M. Simonovits, The mixing rate of Markov chains, an isoperimetric inequality, and computing the volume, 31st IEEE Annual Symposium on Foundations of Computer Science, (1990), 346-354. 
18. L. Lovász and M. Simonovits, Random walks in a convex body and an improved volume algorithm, Random Structures and Algorithms 4 (1993), 359-412.

19. M. Mihail, Conductance and Convergence of Markov Chains: A Combinatorial treatment of Expanders, FOCS (1989), 526-531.

20. O. Perron, Theorie der algebraischen Gleichungen, II (zweite Auflage), de Gruyter, Berlin (1933).

21. D. Spielman and S.-H. Teng, Nearly-linear time algorithms for graph partitioning, graph sparsification, and solving linear systems, Proceedings of the 36th Annual ACM Symposium on Theory of Computing, (2004), 81-90.

22. R. M. Schoen and S. T. Yau, Differential Geometry, International Press, Cambridge, Massachusetts, 1994 . 